\title{
fnálisis del procesamiento de relaciones conceptuales en pacientes con afasia
}

\author{
fnalysis of Conceptual Relation Processing in Aphasic Patients \\ fnálise do processamento de relações conceituais em pacientes com afasia
}

\author{
Leticia Vivas* \\ Conicet-Cimepb/Universidad Nacional de Mar del Plata, Argentina \\ Ricardo García-García, María Victoria Perea-Bartolomé \\ Universidad de Salamanca, España
}

Doi: dx.doi.org/10.12804/apl33.02.2015.10

\section{Resumen}

El daño cerebral producto de un accidente cerebrovascular puede alterar el sistema semántico. Esto se ha observado particularmente en pacientes con afasia. El objetivo de este trabajo es analizar en dichos pacientes el procesamiento de dos tipos de relaciones conceptuales: taxonómicas y temáticas. Se administraron tareas para evaluar la capacidad de establecer y reconocer las relaciones conceptuales mediante vías de presentación verbal y pictórica. Los resultados indicaron que los pacientes con afasia no fluente tienen mayores dificultades para reconocer las relaciones temáticas; mientras que los afásicos fluentes las tienen en tareas que precisan el reconocimiento y el establecimiento de relaciones taxonómicas. Estos resultados apoyan la disociación de los dos tipos de relaciones conceptuales no solo con respecto a sus modos de procesamiento, sino también en su sustrato neuroanatómico. Además, los resultados avalan la disociación entre un sistema conceptual y uno lingüístico. También muestran las diferencias que se pueden observar en función del tipo de tarea (elección libre vs. forzada).

Palabras clave: afasia; relaciones conceptuales; semántica.

\section{flbstract}

Post stroke brain damage can affect the semantic system. This happens particularly in aphasic patients. The aim of this paper is to analyze in such patients the processing of two types of conceptual relations: taxonomic and thematic. We administered a series of tasks to assess the ability to establish and recognize conceptual rela-

* Leticia Vivas, Centro de Procesos Básicos, Metodología y Educación, Universidad Nacional de Mar del Plata, Argentina; Ricardo García-García, Departamento de Psicología Básica, Psicobiología y Metodología de las Ciencias del Comportamiento, Universidad de Salamanca, España; María Victoria Perea-Bartolomé, Departamento de Psicología Básica, Psicobiología y Metodología de las Ciencias del Comportamiento, Universidad de Salamanca, España.

Este trabajo fue posible gracias a una beca otorgada por el Ministerio de Educación de la República Argentina y la Fundación Carolina y al financiamiento de la SeCyT de la Universidad Nacional de Mar del Plata, Argentina.

La correspondencia relacionada con este artículo debe ser enviada a Leticia Vivas, Facultad de Psicología, Universidad Nacional de Mar del Plata, Complejo Universitario Funes 3250, Cuerpo V, Nivel III. Correo electrónico: 1vivas@mdp.edu.ar

Para citar este artículo: Vivas, L., García-García, R. \& Perea-Bartolomé, M. V. (2015). Análisis del procesamiento de relaciones conceptuales en pacientes con afasia. Avances en Psicología Latinoamericana, 33(2), 319-331. doi: dx.doi.org/10.12804/ ap133.02.2015.10 
tions by means of verbal and pictorial presentation. The results indicated that patients with non-fluent aphasia have greater difficulty recognizing the thematic relations, whereas fluent aphasics have greater difficulty in tasks requiring the recognition and establishment of taxonomic relationships. These results support the dissociation of both types of conceptual relations not only with respect to their modes of processing but also in its neuroanatomical substrate. In turn, the results provide information regarding the dissociation between a conceptual and a linguistic system. They also show the difference that can be seen according to the type of task (free choice vs. forced choice).

Key words: aphasia; conceptual relations; semantics.

\section{Resumo}

O dano cerebral produto de um Acidente Vascular Cerebral pode produzir alterações do sistema semântico. Isto tem se observado particularmente em pacientes com afasia. O objetivo deste trabalho é analisar em ditos pacientes o processamento de dois tipos de relações conceituais: taxonômicas e temáticas. Administraram-se uma série de tarefas para avaliar a capacidade de estabelecer e reconhecer as relações conceituais através de vias de apresentação verbal e pictórica. Os resultados indicaram que os pacientes com afasia não fluente têm maiores dificuldades para reconhecer as relações temáticas, enquanto que os afásicos fluentes as têm em tarefas que requerem o reconhecimento e estabelecimento de relações taxonômicas. Estes resultados apoiam a dissociação dos dois tipos de relações conceituais não só com respeito a seus modos de processamento, mas também em seu substrato neuroanatômico. Além disso, os resultados avalizam a dissociação entre um sistema conceitual e um sistema linguístico. Também mostram as diferenças que podem se observar em função do tipo de tarefa (eleição livre vs. Forçada).

Palavras chave: afasia; relações conceituais; semântica.

El estudio de los déficits de categorización semántica en pacientes afásicos resulta de especial interés, de cara a conocer qué tipo de vinculación puede existir entre el procesamiento léxico-semántico y la organización conceptual. Numerosos autores establecen una división teórica entre un nivel de representación conceptual y uno léxico-semántico (Barsalou, Santos, Simmons \& Wilson, 2008; Damasio, Tranel, Grabowski, Adolphs \& Damasio, 2004; Vigliocco \& Filipovic, 2004; Vigliocco \& Vinson, 2007). Los enfoques descomposicionales o de estructura conceptual (Jackendoff, 1995; Moss, Tyler \& Taylor, 2007; Vigliocco \& Filipovic, 2004) consideran que los conceptos están constituidos por un conjunto de rasgos que se encuentran distribuidos y que los rasgos son articulados para los propósitos del lenguaje mediante el sistema léxico-semántico. Según Jackendoff (1995), el contenido semántico del lexicón mental es un subset de estructuras conceptuales que se puede expresar verbalmente. Las representaciones conceptuales forman una interfase entre la información léxica y otros dominios como los sistemas sensoriales y motores. En este sentido, se han descrito en la literatura científica diferentes trabajos, cuya finalidad es conocer si existe una disociación entre ambos sistemas en los pacientes con afasia (Cuetos-Vega \& Castejón, 2005; Robson, Sage \& Lambon Ralph, 2012) o si, por el contrario, hay una asociación entre los déficits observados a nivel lingüístico (particularmente las dificultades en denominación) y un déficit conceptual (Jefferies, Patterson \& Lambon Ralph, 2008; Noonan, Jefferies, Corbett \& Lambon Ralph, 2010; Semenza, Bisiacchi \& Romani, 1992). Un gran número de estudios realizados con pacientes afásicos ha demostrado la presencia de alteraciones léxico-semánticas en los pacientes con afasia de tipo Wernicke (Cuetos-Vega \& Castejón, 2005; Davidoff \& Roberson, 2004; Whitehouse, Caramazza \& Zurif, 1978); sin embargo, son escasos los estudios en los cuales se han estudiado las alteraciones conceptuales en pacientes con afasia de Broca (Hagoort, 1993; Koemeda-Lutz, Cohen \& Meier, 1987).

Dentro del campo de estudio de la categorización semántica, diferentes autores han analizado 
específicamente el procesamiento de las relaciones conceptuales en pacientes con afasias de tipo fluente y de tipo no fluente. Estos incluyeron tareas de procesamiento de relaciones conceptuales taxonómicas y temáticas. Las relaciones taxonómicas son aquellas que vinculan conceptos que forman parte de la misma categoría semántica (e.g. perro-gato) (Lin \& Murphy, 2001); mientras que las relaciones temáticas se definen como relaciones complementarias entre elementos, vivos y no vivos, o eventos que interactúan o coocurren en tiempo y espacio (e. g. perro-correa) (Lin \& Murphy, 2001). Los resultados de estos estudios indican que los pacientes con afasia no fluente, particularmente de tipo Broca, además de presentar un déficit lingüístico, tienen mayores dificultades en establecer relaciones temáticas (Semenza et al., 1992; Semenza, Denes, Lucchese \& Bisiacchi, 1980); entre tanto, los pacientes con afasia fluente, particularmente de tipo Wernicke, aparte de sus déficits lingüísticos, tienen mayores dificultades en establecer relaciones taxonómicas (Cuetos-Vega \& Castejón, 2005; Davidoff \& Roberson, 2004; Gardner \& Zurif, 1976; Melice-Ledent, Gainotti, Masserli \& Tissot, 1976; Semenza et al., 1980). Sin embargo, también hay estudios que no han encontrado dificultades semánticas en pacientes con afasia no fluente (la mayoría de ellos diagnosticados con afasia de tipo Broca) en comparación con pacientes con afasia fluente (McCleary \& Hirst, 1986; Wayland \& Taplin, 1982).

Aunque numerosos estudios sostienen que los pacientes con afasia fluente presentan afectación en el procesamiento semántico, no está claramente definida la presencia de afectación semántica en aquellos casos de afasia no fluente. Por lo tanto, tampoco está establecido si hay una vinculación entre los déficits lingüísticos y los conceptuales en estos pacientes. Es probable que esto se deba, al menos en parte, a que en la evaluación de los pacientes con afasia no fluente se suele poner el acento en las dificultades lingüísticas (más predominantes), más que en las conceptuales, con lo cual no se suelen incluir tareas para evaluar distin- tos tipos de relaciones conceptuales por distintas vías de presentación. A su vez, en muchos de los trabajos citados previamente las pruebas utilizadas consisten en tareas de elección forzada, es decir, tareas en las que se presenta un número limitado de opciones de respuesta y la persona debe hacer su elección entre ellas. Las tareas de elección libre, por el contrario, al no presentar opciones de respuesta, brindan la posibilidad de valorar la preferencia que tiene el paciente por un tipo u otro de relación conceptual. Consideramos que podría ser de especial interés el incluir en la evaluación tareas de elección libre, las cuales facilitarían el análisis sobre el tipo de relación que establece espontáneamente el paciente frente a los estímulos presentados.

En función de lo expuesto, el presente trabajo tiene como propósito estudiar cómo se establecen las relaciones conceptuales taxonómicas y temáticas en pacientes con diferentes tipos de trastorno afásico (fluente y no fluente) en comparación con participantes sin afectación neurológica. Se analizará el impacto de las vías de presentación de los estímulos (verbal vs. pictórica) y el formato de la tarea: de elección forzada (que tengan un solo tipo de respuesta correcta) o de respuesta libre (donde el paciente proponga su criterio de organización del material).

\section{Método}

\section{Participantes}

Se evaluaron 25 pacientes con afasia que habían sufrido un accidente cerebro-vascular (ACV) atendidos en el Hospital Privado de Comunidad (HPC) y el Hospital Interzonal General de Agudos (HIGA) Oscar Alende, de la ciudad de Mar del Plata (Argentina). Los pacientes participaron de forma voluntaria en el estudio con consentimiento informado. Este estudio, así como el formulario de consentimiento informado, fue aprobado por el Comité de Ética del Comité Institucional de Revisión de Estudios de Investigación del HPC y 
el Comité de Investigación del HIGA. Este estudio se realizó siguiendo las normas éticas establecidas en la Declaración de Helsinki. Los criterios de inclusión para los pacientes fueron: (a) haber sufrido una lesión cerebral focal como consecuencia de un ACV; (b) estar orientado en tiempo, espacio y persona; (c) no estar sometidos a respiración artificial; (d) no poseer indicios de diagnóstico clínico de demencia o déficit cognitivo previo de acuerdo con la evaluación del neurólogo y los datos de la historia clínica; (e) no poseer historia previa de diagnóstico de afectación psicopatológica relevante o parcialmente incapacitante; (f) tener visión normal o corregida a normal, y (g) comprender órdenes simples. La presencia de afasia severa fue criterio de exclusión.

El grupo de pacientes fue subdividido de acuerdo con el tipo de afasia. Para ello se administró un instrumento argentino, el Test de Evaluación Breve de las Afasias (Vigliecca et al., 2011) en el momento de realizar la evaluación neuropsicológica. Los pacientes fueron clasificados en dos grupos: afásicos fluentes y no fluentes de acuerdo con los criterios de Helo (2007) y Toledo (2007). En este sentido, los pacientes con afasia de Wernicke y transcortical sensorial (Benson \& Ardila, 1996) se ubicaron dentro del grupo de las afasias fluentes y los pacientes con afasia de Broca y transcortical motora (Benson \& Ardila, 1996) se ubicaron dentro del grupo de no fluentes. De este modo quedaron conformados dos grupos de pacientes: (a) con afasia fluente $(N=7)$ y (b) con afasia no fluente $(N=$ 18). Las características de las lesiones y tipo de afasia de los pacientes se detallan en el apéndice 1. Todos los pacientes presentaron lesiones izquierdas.

A su vez, se evaluaron 30 participantes controles sin afectación neurocognitiva pareados por edad, género y nivel de escolarización con los pacientes. Estos participantes se seleccionaron mediante un muestreo intencional de acuerdo con las características del grupo clínico y participaron de forma voluntaria en el estudio. Los criterios de inclusión para el grupo control fueron: (a) tener una puntua- ción en el MMSE (Butman et al., 2001; Folstein, Folstein \& McHugh, 1975) superior a 26 puntos; (b) tener una puntuación en la Escala de Ansiedad y Depresión de Goldberg (Goldberg, Bridges, Duncan-Jones, \& Grayson, 1988; Montón et al., 1993) menor a 5 en la subescala de ansiedad y a 3 en la de depresión; (c) no poseer historia clínicamente demostrable de afectación neurológica, psiquiátrica o neuropsicológica; (d) no poseer historia previa de alcoholismo u otras tóxico-dependencias, y (e) no estar bajo tratamiento con antidepresivos o ansiolíticos en la actualidad. Los datos demográficos de los pacientes y de los controles sanos se describen en la tabla 1.

Tabla 1

Datos demográficos

\begin{tabular}{|c|c|c|c|c|}
\hline & & Edad & Escolaridad & Género \\
\hline \multirow{6}{*}{ 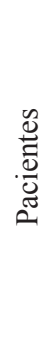 } & \multirow{3}{*}{ Fluentes } & \multirow{3}{*}{$\begin{array}{l}75.83(D S \\
7.441)\end{array}$} & $61 \%$ primario & \multirow{3}{*}{$42 \% \mathrm{~F} / 58 \% \mathrm{M}$} \\
\hline & & & $28 \%$ secundario & \\
\hline & & & $\begin{array}{l}7 \% \text { terciario/ } \\
\text { universitario }\end{array}$ & \\
\hline & \multirow{3}{*}{$\begin{array}{l}\text { No } \\
\text { fluentes }\end{array}$} & & $57 \%$ primario & \multirow{3}{*}{$38 \% \mathrm{~F} / 62 \% \mathrm{M}$} \\
\hline & & $78.87(D S$ & $32 \%$ secundario & \\
\hline & & $6.289)$ & $\begin{array}{l}7 \% \text { terciario/ } \\
\text { universitario }\end{array}$ & \\
\hline \multirow{3}{*}{\multicolumn{2}{|c|}{ Controles }} & & $65 \%$ primario & \multirow{3}{*}{$59 \% \mathrm{~F} / 41 \% \mathrm{M}$} \\
\hline & & $70.04(D S$ & $26 \%$ secundario & \\
\hline & & 15.428) & $\begin{array}{l}8.7 \% \text { terciario/ } \\
\text { universitario }\end{array}$ & \\
\hline
\end{tabular}

F: femenino; M: masculino.

\section{Instrumentos}

Se administraron las siguientes pruebas para verificar el cumplimiento de los criterios de inclusión: Escala de Ansiedad y Depresión de Goldberg (Goldberg et al., 1988; Montón et al., 1993); versión abreviada del Test del Informador (Morales González, González-Montalvo, Del Ser Quijano \& Bermejo Pareja, 1992); Prueba Emparejamiento Palabra Oída-Dibujo y de Denominación de la batería EMSDA (Peraita Adrados, González Labra, Sánchez Bernardos \& Galeonte, 2000); Prueba de 
Comprensión de Órdenes Complejas de la Batería Barcelona (Peña-Casanova, 2005). A su vez, se administró el Test de Evaluación Breve de las Afasias (Vigliecca et al., 2011) para determinar el tipo de afasia.

Para evaluar las relaciones conceptuales se administraron dos tareas de respuesta cerrada, una para relaciones temáticas (Test de Pirámides y Faraones) y una para relaciones taxonómicas, ambas en formato verbal y pictórico, y dos tareas de respuesta abierta, que permiten valorar el tipo de relación que elige libremente la persona (Clasificación de Dibujos y DISTSEM), también en formato verbal y pictórico. Las tareas se detallan a continuación.

\section{Prueba de relaciones temáticas, Pirámides y} Faraones en versión verbal y pictórica. Se trata de una adaptación argentina abreviada realizada por Martínez-Cuitiño y Barreiro (2010) de la Prueba de Pirámides y Palmeras de Howard y Patterson (1992). Esta prueba permite evaluar la capacidad de detectar relaciones temáticas. El formato de presentación es del tipo emparejamiento-con-la-muestra (matching-to-sample), en el cual se presentan dos palabras (o dibujos en la versión pictórica) debajo y una arriba. El paciente debe decidir con cuál de las dos de abajo se asocia más la palabra (imagen) de arriba. La prueba consta de 19 ítems. La administración fue realizada mediante el Presentation Software 10.1 (Neurobehavioral Systems, disponible en http://www.neurobs.com/) para poder medir los tiempos de respuesta. En su versión argentina esta prueba tiene una especificidad del $98.8 \%$ y una sensibilidad del $85 \%$ para detectar individuos con dificultades semánticas.

Tarea de relaciones taxonómicas verbal y pictórica. Esta prueba se elaboró en un trabajo previo (Vivas, 2012) con la finalidad de obtener una medida del reconocimiento de las relaciones taxonómicas siguiendo el formato de presentación y la consigna de la prueba de Pirámides y Palmeras.
Las tríadas estuvieron conformadas por palabras o dibujos pertenecientes a la misma categoría semántica, pero con distinta proximidad. Por ejemplo, dentro de la misma categoría semántica águila y pato tienen mayor proximidad que águila y rinoceronte. Quedaron conformados en la versión final 18 ítems (véase el apéndice 2) y se obtuvo un número de ítems equivalente entre la prueba de Pirámides y Faraones y la tarea de relaciones taxonómicas. Se escogió el mismo número de ítems para cada categoría semántica. La consigna solicitaba responder con cuál de los dos ítems de abajo se asocia más el de arriba. Esta consigna es la misma que la utilizada en la versión argentina de la prueba de Pirámides y Faraones. La tarea se administró mediante el Presentation Software.

Prueba de Clasificación de Dibujos de la batería EMSDA (Peraita Adrados et al., 2000) para evaluar los criterios de agrupamientos de imágenes. Consiste en la presentación de 18 dibujos pertenecientes a las categorías alimentos, prendas de vestir, muebles, animales, medios de transporte y plantas que, a su vez, cuentan con posibles vinculaciones temáticas. La persona debe organizarlos formando grupos siguiendo el criterio que quiera. Se solicita a la persona que justifique su elección. Los criterios utilizados fueron clasificados en taxonómico, temático u otros. Si la persona falla en más de 4 elementos se administra la versión dirigida, donde el experimentador suministra previamente las categorías y el sujeto debe ubicar los objetos en la categoría que corresponde.

Método DISTSEM (Vivas, J., 2008; Vivas, L., 2010). Permite evaluar la red semántica correspondiente a un conjunto de conceptos basándose en las estimaciones de proximidad realizadas por los sujetos entre pares de palabras y de distinta categoría semántica. El método consta de 21 ítems (véase apéndice 3). Las respuestas se registran mediante una escala Likert con cinco opciones, donde el valor más bajo representaba la ausencia de propie- 
dades en común entre los conceptos y el más alto la presencia de muchas propiedades en común. También se toma registro del criterio que se utiliza para estimar las proximidades entre pares de palabras.

\section{Procedimiento}

Después de establecer el consentimiento informado, se evaluó individualmente a cada paciente mediante las pruebas anteriormente detalladas. La administración de los instrumentos en el grupo de pacientes demandó dos sesiones de evaluación individuales de aproximadamente 40 minutos. La evaluación se realizó un mes después del alta hospitalaria. Se adoptó la temporalidad de un mes, porque transcurrido ese periodo los síntomas aún presentan vivacidad y aún no se han desarrollado mecanismos compensatorios del déficit que pueden interferir en la interpretación del desempeño (Basso \& Pizzamiglio, 1999; Voytek et al., 2010).

El grupo control fue seleccionado mediante un muestreo intencional siguiendo las características sociodemográficas del grupo de pacientes. Una vez establecido el consentimiento informado y verificado que cumplieran con los criterios de inclusión, se administraron las pruebas en dos entrevistas de 30 minutos aproximadamente.
Las variables dependientes de interés fueron el desempeño en el reconocimiento y establecimiento de relaciones conceptuales taxonómicas y temáticas, y las variables independientes, la presencia o ausencia de afasia y el tipo (fluente o no fluente). Fueron evaluadas mediante las tareas mencionadas.

\section{Resultados}

Se establecieron cuatro grupos de análisis de acuerdo con la presencia o ausencia de patología, la presencia de afasia y el tipo de afasia (fluente y no fluente). Dado que la distribución de los datos no cumplía con la normalidad estadística de acuerdo con el método de Kolmogorov-Smirnov $(p<.05)$, se escogieron pruebas no paramétricas. Se aplicó la prueba U de Mann-Whitney mediante el paquete estadístico SPSS versión 17 para analizar si había diferencias entre los grupos establecidos en todas las pruebas de relaciones conceptuales. Para realizar la comparación post hoc entre los pares de grupos se utilizó el método sugerido por Sprent y Smeeton (2007). En la tabla 2 pueden observarse los valores obtenidos en todas las tareas para cada grupo de sujetos.

En la tabla 3 se detallan los resultados de la prueba de Kruskal-Wallis y nivel de significación

Tabla 2

Estadísticos para cada tarea por grupo

\begin{tabular}{|c|c|c|c|c|c|c|c|c|}
\hline \multicolumn{3}{|c|}{ Tipo de afasia } & $\begin{array}{l}\text { Z Taxón }{ }^{\text {a }} \\
\text { verbal }\end{array}$ & $\begin{array}{l}\mathrm{ZPyF}_{-} \mathrm{Py}^{-} \\
\text {verbal }\end{array}$ & $\begin{array}{l}\text { Z Taxón- } \\
\text { pictórico }\end{array}$ & $\begin{array}{l}\text { Z PyF- } \\
\text { pictórico }\end{array}$ & $\begin{array}{l}\text { Z clasificación } \\
\text { de dibujos }\end{array}$ & Z DISTSEM \\
\hline \multirow{4}{*}{ Control } & \multirow{2}{*}{$N$} & Válidos & 30 & 30 & 30 & 30 & 30 & 30 \\
\hline & & Perdidos & 0 & 0 & 0 & 0 & 0 & 0 \\
\hline & \multicolumn{2}{|c|}{ Moda } & -.386 & .156 & .950 & .769 & .233 & .960 \\
\hline & \multicolumn{2}{|c|}{ Rango } & 3.090 & 3.162 & 3.462 & 4.096 & 4.693 & 3.286 \\
\hline \multirow{4}{*}{ No fluente } & \multirow{2}{*}{$N$} & Válidos & 16 & 16 & 18 & 18 & 18 & 13 \\
\hline & & Perdidos & 2 & 2 & 0 & 0 & 0 & 5 \\
\hline & \multicolumn{2}{|c|}{ Moda } & $-2.699^{a}$ & $-9.329^{\mathrm{a}}$ & .950 & -1.206 & .233 & $-9.503^{\mathrm{a}}$ \\
\hline & \multicolumn{2}{|c|}{ Rango } & 5.405 & 10.541 & 6.226 & 11.845 & 7.478 & 9.683 \\
\hline
\end{tabular}




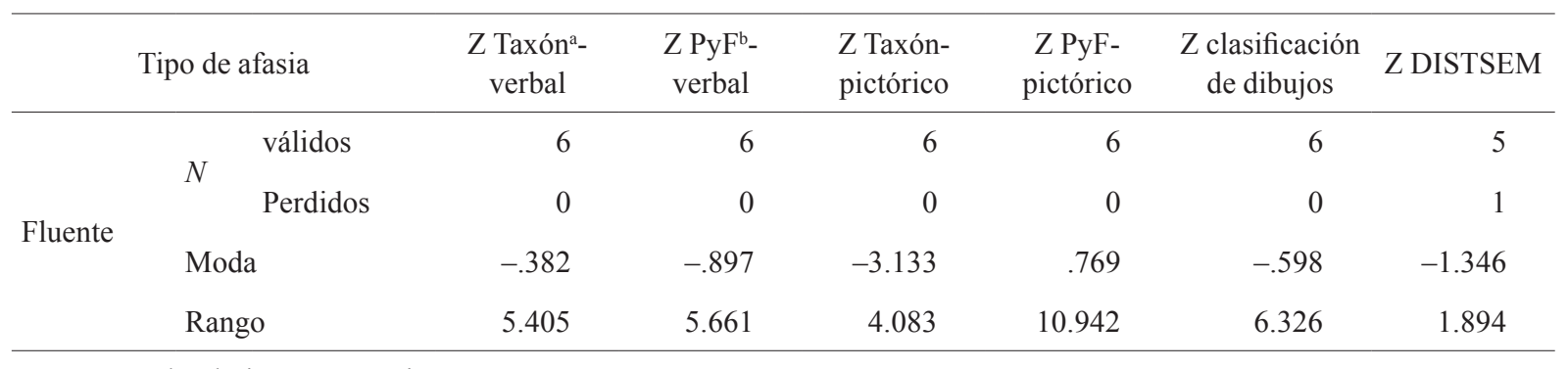

Taxón: tarea de relaciones taxonómicas.

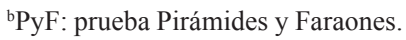

Tabla 3

Prueba de Kruskal-Wallis para las seis tareas

\begin{tabular}{|c|c|c|c|c|c|c|}
\hline & Taxón ${ }^{\mathrm{a}}$-verbal & $\mathrm{PyF}^{\mathrm{b}}$-verbal & Taxón-pictórico & PyF-pictórico & DISTSEM & Clasificación de dibujos \\
\hline Chi-cuadrado & 14.254 & 13.432 & 4.176 & 11.499 & 15.590 & 6.241 \\
\hline Gl & 2 & 2 & 2 & 2 & 2 & 2 \\
\hline Sig. asint. & .001 & .001 & .124 & .033 & .000 & .044 \\
\hline
\end{tabular}

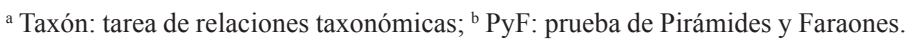

al comparar las puntuaciones obtenidas por cada uno de los grupos (afásicos, fluentes y no fluente, y controles) en cada una de las tareas de relaciones conceptuales.

Se puede observar que los grupos presentaron diferencias significativas en todas las tareas, menos en la tarea de relaciones taxonómicas por vía pictórica. En la tabla 4 se observan los valores de $p$ obtenidos para cada par de grupos de acuerdo con el análisis post hoc.

Los pacientes con afasia no fluente presentaron diferencias altamente significativas con el grupo control en todas las tareas menos en Clasificación de Dibujos, donde hubo una diferencia con un nivel de significación más moderado $(p<.05)$. Por su parte, el grupo de pacientes con afasia fluente presentó diferencias altamente significativas con el grupo control en la tarea verbal de relaciones taxonómicas, la tarea verbal de relaciones temáticas y Clasificación de Dibujos y una diferencia significativa pero más moderada en la tarea de estimación de distancias semánticas (DISTSEM) y sin diferencias significativas en la tarea pictórica de relaciones temáticas.
Tabla 4

Valores de significación del análisis post hoc

\begin{tabular}{|c|c|c|c|}
\hline Tarea & Grupos & & $p$ \\
\hline \multirow{3}{*}{ 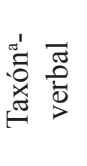 } & Control & Afasia no fluente & $.000^{* *}$ \\
\hline & & Afasia fluente & $.002^{* *}$ \\
\hline & Afasia no fluente & Afasia fluente & .441 \\
\hline \multirow{3}{*}{ 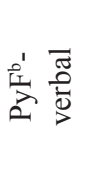 } & Control & Afasia no fluente & $.000^{* *}$ \\
\hline & & Afasia fluente & $.006^{* *}$ \\
\hline & Afasia no fluente & Afasia fluente & .334 \\
\hline \multirow{3}{*}{ 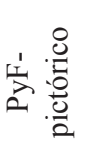 } & Control & Afasia no fluente & $.002^{* *}$ \\
\hline & & Afasia fluente & .060 \\
\hline & Afasia no fluente & Afasia fluente & .291 \\
\hline \multirow{3}{*}{ 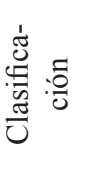 } & Control & Afasia no fluente & $.050^{*}$ \\
\hline & & Afasia fluente & $.002^{* *}$ \\
\hline & Afasia no fluente & Afasia fluente & .066 \\
\hline \multirow{3}{*}{ 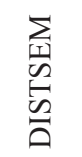 } & Control & Afasia no fluente & $.000^{* *}$ \\
\hline & & Afasia fluente & $.016^{*}$ \\
\hline & Afasia no fluente & Afasia fluente & .197 \\
\hline
\end{tabular}

*Significativo al $.05 ; * *$ significativo al .01 .

a Taxón: tarea de relaciones taxonómicas; ${ }^{b}$ PyF: prueba de Pirámides y Palmeras. 


\section{Discusión}

En primer lugar, los resultados obtenidos indican que ambos grupos de pacientes afásicos tuvieron puntuaciones más bajas que los controles en las tareas verbales de elección forzada (Taxón y PyF), lo cual corrobora una afectación del sistema lingüístico. Sin embargo, uno de los hallazgos de mayor interés en este estudio es el hecho de que estos pacientes también tuvieron dificultades en las tareas pictóricas, y ello evidencia la presencia de déficits conceptuales no verbales. En la literatura científica se ha discutido y constatado esta distinción entre dificultades lingüísticas y conceptuales en pacientes con afasia pos-ACV (Cuetos-Vega \& Castejón, 2005) y revisiones recientes indican que son altamente frecuentes las dificultades conceptuales no verbales en pacientes con afasia (Gainotti, 2014). Uno de los aportes de mayor relevancia del presente trabajo es que los resultados brindan evidencia sobre las características diferenciales de las dificultades conceptuales no verbales de acuerdo con el tipo de afasia.

Ambos grupos de pacientes con afasia presentaron patrones diferentes con respecto al grupo control. Se puede ver que los pacientes con afasia no fluente tienen más dificultades en el establecimiento de relaciones temáticas con respecto al grupo control; mientras que los pacientes con afasia fluente tienen dificultades particularmente en el establecimiento de relaciones taxonómicas. Estos resultados van en el mismo sentido que los obtenidos por Cuetos-Vega y Castejón (2005), Davidoff y Roberson (2004), Gardner y Zurif (1976), Melice-Ledent et al. (1976) y Semenza et al. (1980), quienes afirmaron que los pacientes con afasia de Wernicke (afasia de tipo fluente) suelen tener mayores dificultades en el establecimiento de relaciones taxonómicas y los pacientes con afasia de Broca (afasia de tipo no fluente) suelen tener mayores dificultades en el establecimiento de relaciones temáticas. Esto podría deberse a que el procesamiento de las relaciones taxonómicas y te- máticas se realiza en áreas cerebrales parcialmente independientes y por eso se ven afectadas de manera diferencial en ambos tipos de afasia. Algunos estudios demuestran que el procesamiento de las primeras requiere la activación de áreas cerebrales posteriores, pues las relaciones de este tipo necesitan buscar similitudes perceptivas (particularmente visuales) (Kalènine et al., 2009). Por su parte, las segundas implican situar a los elementos en un mismo contexto y están más vinculadas a la activación de áreas temporo-parietales (Kalènine et al., 2009). No hay evidencia hasta el momento, sin embargo, de que el procesamiento de las relaciones temáticas implique específicamente el área de Broca (para una revisión del tema véase Vivas, 2012).

Los datos obtenidos en este trabajo también constituyen un aporte a los modelos de procesamiento conceptual, ya que indican que ambos tipos de relaciones, taxonómicas y temáticas pueden verse afectadas de manera diferencial, lo cual sugiere que tienen cierta independencia, tal como proponen trabajos previos sobre el tema (Kalénine et al., 2009; Lin \& Murphy, 2001; Sachs et al., 2008).

Los resultados del presente trabajo sugieren la existencia de diferencias en el procesamiento de relaciones conceptuales en los pacientes con afasia fluente y no fluente y apoyan la idea de que existen dos sistemas de procesamiento independiente, uno lingüístico y uno conceptual, y que si bien el primero estaría afectado en ambos tipos de afasia, el segundo se afectaría de manera diferencial en cada una.

Finalmente, cabe mencionar algunas de las limitaciones del presente trabajo. En primer lugar, para evaluar las relaciones temáticas se utilizó una prueba reconocida de evaluación de la memoria semántica (prueba de Pirámides y Faraones); mientras que en las relaciones taxonómicas se diseñó una tarea experimental equivalente, dada la inexistencia de una prueba reconocida para evaluar este tipo de relaciones. En estudios futuros sería deseable poder contar con dos tareas equivalentes para evaluar ambos tipos de relaciones conceptua- 
les con un riguroso control de la fuerza asociativa entre los estímulos presentados. A su vez, si bien en este estudio se utilizaron dos tareas de elección libre disponibles, también sería de interés poder contar con una tarea libre con presentación verbal y pictórica en la cual los estímulos puedan ser agrupados tanto taxonómica como temáticamente, a fin de poder observar el criterio preponderante utilizado por el paciente.

\section{Referencias}

Barsalou, L., Santos, A., Simmons, W. K. \& Wilson, C. (2008). Language and simulation in conceptual processing. En Symbols, embodiment, and meaning (pp. 245-283). Oxford: Oxford University Press.

Basso, A. \& Pizzamiglio, L. (1999). Recovery of cerebral functions. En Handbook of clinical and experimental neuopsychology (pp. 849-868). London: Psychology Press.

Benson, D. F. \& Ardila, A. (1996). Aphasia: A clinical perspective. Oxford: Oxford University Press.

Butman, J., Arizaga, R., Harris, P., Drake, M., Baumann, D., de Pascale, A. et al. (2001). El "Mini-Mental State Examination" en español: normas para Buenos Aires. Revista Neurológica Argentina, 26(1), 11-15.

Cuetos-Vega, F. \& Castejón, L. (2005). Disociación de la información conceptual y lingüística a partir de un estudio de caso. Revista de Neurología, 8(41), 469-474.

Damasio, H., Tranel, D., Grabowski, T., Adolphs, R. \& Damasio, A. (2004). Neural systems behind word and concept retrieval. Cognition, 92(1-2), 179-229. doi:10.1016/j.cognition.2002.07.001

Davidoff, J. \& Roberson, D. (2004). Preserved thematic and impaired taxonomic categorisation: A case study. Language and Cognitive Processes, 19(1), 137-174.

Folstein, M. F., Folstein, S. E. \& McHugh, P. R. (1975). "Mini-mental state": A practical method for grading the cognitive state of patients for the clinician. Journal of Psychiatric Research, 12(3), 189-198.

Gainotti, G. (2014). Old and recent approaches to the problem of non-verbal conceptual disorders in aphasic patients. Cortex, 1130. doi: 10.1016/j. cortex.2014.01.009

Gardner, H. \& Zurif, E. (1976). Critical reading of words and phrases in aphasia. Brain and Language, 3(2), 173-190. doi: 10.1016/0093934X(76)90015-8

Goldberg, D., Bridges, K., Duncan-Jones, P. \& Grayson, D. (1988). Detecting anxiety and depression in general medical settings. BMJ: British Medical Journal, 297(6653), 897-899.

Hagoort, P. (1993). Impairments of lexical-semantic processing in aphasia: evidence from the processing of lexical ambiguities. Brain and Language, 45(2), 189-232. doi: 10.1006/brln.1993.1043

Helo, A. (2007). Diagnóstico diferencial de las afasias fluentes. En Diagnóstico diferencial de las afasias (pp. 14-16). Santiago: Universidad de Chile.

Howard, D. \& Patterson, K. (1992). The Pyramids and Palm Trees test. London: Pearson.

Jackendoff, R. (1995). Languages of the mind: Essays on mental representation. Boston: MIT Press.

Jefferies, E., Patterson, K. \& Lambon Ralph, M. A. (2008). Deficits of knowledge vs. executive control in semantic cognition: Insights from cued naming. Neuropsychologia, 46(2), 649-658. doi: 10.1016/j.neuropsychologia.2007.09.007

Kalénine, S., Peyrin, C., Pichat, C., Segebarth, C., Bonthoux, F. \& Baciu, M. (2009). The sensory-motor specificity of taxonomic and thematic conceptual relations: a behavioral and fMRI study. NeuroImage, 44(3), 1152-1162. doi: 10.1016/j.neuroimage.2008.09.043

Koemeda-Lutz, M., Cohen, R. \& Meier, E. (1987). Organization of and access to semantic memory in aphasia. Brain and Language, 30(2), 321-337. 
Lin, E. \& Murphy, G. (2001). Thematic relations in adults' concepts. Journal of Experimental Psychology, 1(130), 3-28.

Martínez-Cuitiño, M. \& Barreiro, J. (2010). ¿Pirámides y Palmeras o Pirámides y Faraones?: Adaptación y validación de un test de asociación semántica al español rioplatense. Interdisciplinaria, 2(27), 247-260.

McCleary, C. \& Hirst, W. (1986). Semantic classification in aphasia: a study of basic, superordinate, and function relations. Brain and Language, 27(2), 199-209.

Melice-Ledent, S., Gainotti, G., Masserli, P. \& Tissot, R. (1976). Logique elementaire et champs semantiques dans l'aphasie. Revue Neurologique, 132, 343-359.

Montón, C., Pérez Echeverría, M., Campos, R., García Campayo, J., Lobo, A., El Gzempp et al. (1993). Escalas de ansiedad y depresión de Goldberg: una guía de entrevista eficaz para la detección del malestar psíquico. Atención Primaria, 6(12), 345-349.

Morales González, J. M., González-Montalvo, J. I., Del Ser Quijano, T. \& Bermejo Pareja, F. (1992). [Validation of the S-IQCODE: the Spanish version of the informant questionnaire on cognitive decline in the elderly]. Archivos de Neurobiologiá, 55(6), 262-266.

Moss, H. E., Tyler, L. K. \& Taylor, K. I. (2007). Conceptual structure. En The Oxford handbook of psycholinguistics (pp. 217-234). Oxford: Oxford University Press.

Noonan, K. A., Jefferies, E., Corbett, F. \& Lambon Ralph, M. A. (2010). Elucidating the nature of deregulated semantic cognition in semantic aphasia: evidence for the roles of prefrontal and temporo-parietal cortices. Journal of Cognitive Neuroscience, 22(7), 1597-1613. doi: 10.1162/ jocn.2009.21289

Peña-Casanova, J. (2005). Programa integrado de exploración neuropsicológica. Barcelona: Masson.

Peraita Adrados, H., González Labra, M., Sánchez Bernardos, M. \& Galeonte, M. (2000). Bate- ría de evaluación del deterioro de la memoria semántica en Alzheimer. Psicothema, 2(12), 192-200.

Robson, H., Sage, K. \& Lambon Ralph, M. A. (2012). Wernicke's aphasia reflects a combination of acoustic-phonological and semantic control deficits: a case-series comparison of Wernicke's aphasia, semantic dementia and semantic aphasia. Neuropsychologia, 50(2), 266-275. doi: 10.1016/j.neuropsychologia.2011.11.021 Sachs, O., Weis, S., Zellagui, N., Huber, W., Zvyagintsev, M., Mathiak, K. \& Kircher, T. (2008). Automatic processing of semantic relations in fMRI: Neural activation during semantic priming of taxonomic and thematic categories. Brain Research, 1218, 194-205. doi: 10.1016/j. brainres.2008.03.045

Semenza, C., Bisiacchi, P. S. \& Romani, L. (1992). Naming disorders and semantic representations. Journal of Psycholinguistic Research, 21(5), 349-364.

Semenza, C., Denes, G., Lucchese, D. \& Bisiacchi, P. (1980). Selective deficit of conceptual structures in aphasia: class versus thematic relations. Brain and Language, 10(2), 243-248.

Sprent, P. \& Smeeton, N. (2007). Applied nonparametric statistical methods. Boca Raton, Florida: Chapman \& Hall/CRC.

Toledo, L. (2007). Diagnóstico diferencial de las afasias no fluentes. En Diagnóstico diferencial de las afasias (pp. 17-20). Santiago: Universidad de Chile.

Vigliecca, N. S., Peñalva, M. C., Castillo, Molina, S. C., Voos, J. A., Ortiz, M. et al. (2011). Brief aphasia evaluation (minimum verbal performance): concurrent and conceptual validity study in patients with unilateral cerebral lesions. Brain Injury: [BI], 25(4), 394-400. doi:10.3109/026 99052.2011 .556106

Vigliocco, G. \& Filipovic, L. (2004). From mind in the mouth to language in the mind. Trends in Cognitive Sciences, 8(1), 5-7. doi: 10.1016/j. tics.2003.10.018 
Vigliocco, G. \& Vinson, D. P. (2007). Semantic representation. En The Oxford handbook of psycholinguistics (pp. 195-215). Oxford: Oxford University Press. Recuperado de http://homepage.psy.utexas.edu/HomePage/Faculty/Griffin/ independent/Vigliocco_Vinson2007_Semantics_chapter.pdf

Vivas, J. (2008). DISTSEM: un método de captura y graficación de redes semánticas. Aplicaciones a Educación y a Neuropsicología. Revista Mexicana de Psicología, 27-31.

Vivas, L. Y. (2010). Aplicación de un método para el análisis de las redes semánticas en pacientes que sufrieron un accidente cerebro vascular. Interdisciplinaria, 27(1), 147-162.
Vivas, L. Y. (2012). Procesamiento de relaciones conceptuales en pacientes con lesiones neurológicas focales. Doctoral Thesis. Recuperado de http://gredos.usal.es/jspui/handle/10366/115634

Voytek, B., Davis, M., Yago, E., Barcelo, F., Vogel, E. K. \& Knight, R. T. (2010). Dynamic neuroplasticity after human prefrontal cortex damage. Neuron, 68(3), 401-408. doi: 10.1016/j. neuron.2010.09.018

Wayland, S. \& Taplin, J. E. (1982). Nonverbal categorization in fluent and nonfluent anomic aphasics. Brain and Language, 16(1), 87-108.

Whitehouse, P., Caramazza, A. \& Zurif, E. (1978). Naming in aphasia: interacting effects of form and function. Brain and Language, 6(1), 63-74.
Fecha de recepción: 16 de octubre de 2013 Fecha de aceptación: 1 de diciembre de 2014 


\begin{tabular}{|c|c|c|c|c|c|c|}
\hline & & Cara & $\begin{array}{r}\text { Apénd } \\
\text { cterísticas de las le }\end{array}$ & $\begin{array}{l}\text { ice } 1 \\
\text { siones y tipo de }\end{array}$ & afasia & \\
\hline Pacientes & Estudio & Lateralización & Localización & Córtical-subcortical & Tipo de ACV & Afasia \\
\hline AM & TAC & Izquierda & SD & Cortico-subcortical & Hemorrágico & No fluente \\
\hline ASC & TAC & Izquierda & Parietal & Cortical & Hemorrágico & No fluente \\
\hline ASI & $\mathrm{RMN}$ & Izquierda & Frontal & Cortical & Isquémico & No fluente \\
\hline BS & TAC & Izquierda & Occipital & Cortical & Hemorrágico & No fluente \\
\hline $\mathrm{BZ}$ & $\mathrm{RMN}$ & Izquierda & SD & Córtico-subcortical & Isquémico & No fluente \\
\hline $\mathrm{DE}$ & TAC & Izquierda & $\mathrm{SD}$ & SD & Isquémico & No fluente \\
\hline EAL & TAC & Izquierda & SD & SD & Isquémico & No fluente \\
\hline $\mathrm{EC}$ & TAC & Izquierda & $\mathrm{SD}$ & Subcortical & Isquémico & Fluente \\
\hline ELE & $\mathrm{RMN}$ & Izquierda & Frontal & Córtico-subcortical & Isquémico & No fluente \\
\hline ELU & TAC & Izquierda & Frontotemporal & Córtico-subcortical & Isquémico & No fluente \\
\hline FR & TAC & Izquierda & Frontroparietal & Subcortical & Hemorrágico & Fluente \\
\hline FT & TAC & Izquierda & Temporoparietooccipital & & Isquémico & Fluente \\
\hline GG & TAC & Izquierda & $\mathrm{SD}$ & Subcortical & Isquémico & No fluente \\
\hline JP & TAC & Izquierda & Ganglios de la base & $\mathrm{SD}$ & Isquémico & No fluente \\
\hline JS & TAC & Izquierda & Frontoparietal & Subcortical & Isquémico & Fluente \\
\hline LS & TAC & Izquierda & Capsular & Cortical & Isquémico & No fluente \\
\hline $\mathrm{MC}$ & $\mathrm{RMN}$ & Izquierda & Parietooccipital & Córtico-subcortical & $\begin{array}{l}\text { Isquémico con transfor- } \\
\text { mación hemorrágica }\end{array}$ & Fluente \\
\hline MPR & $\mathrm{RMN}$ & Izquierda & SD & Córtico-subcortical & Isquémico & No fluente \\
\hline MR & TAC & Izquierda & Frontoparietal & Cortical & Isquémico & No fluente \\
\hline NA & $\mathrm{RMN}$ & Izquierda & Temporoparietal & Córtico-subcortical & Isquémico & No fluente \\
\hline RA & $\mathrm{RMN}$ & Izquierda & Frontal & Cortical & Isquémico & No fluente \\
\hline SR & TAC & Izquierda & $\mathrm{SD}$ & SD & Isquémico & Fluente \\
\hline $\mathrm{TM}$ & TAC & Izquierda & Frontoparietal & Cortical & Isquémico & No fluente \\
\hline VC & TAC & Izquierda & Tálamo & Subcortical & Hemorrágico & No fluente \\
\hline VP & TAC & Izquierda & SD & Subcortical & Isquémico & Fluente \\
\hline
\end{tabular}

SD: sin dato; TAC: tomografía axial computarizada; RMN: resonancia magnética nuclear. 
Apéndice 2

\section{Estímulos que conforman la tarea de relaciones taxonómicas}

\begin{tabular}{lll}
\hline \multicolumn{1}{c}{ Estímulo de referencia } & \multicolumn{1}{c}{ Estímulo objetivo } & \multicolumn{1}{c}{ Estímulo distractor } \\
\hline vestido & pollera (falda en España) & corbata \\
cómoda & escritorio & sillón \\
zanahoria & cebolla & banana \\
águila & pato & rinoceronte \\
camión & bicicleta & velero \\
peine & cepillo & tenedor \\
pantalón & saco & gorra \\
banco & mecedora & cama \\
pera & manzana & limón \\
caballo & cebra & león \\
colectivo & moto & helicóptero \\
hacha & serrucho & destornillador \\
saco & blusa & pollera (falda en España) \\
choclo & tomate & uvas \\
león & gato & chancho \\
avión & helicóptero & tractor \\
banquito & silla & cama \\
lápiz & tijera & pincel \\
\hline
\end{tabular}

Apéndice 3

Pares de estímulos de la prueba DISTSEM

Naranja-manzana

Chancho*-silla

Mesa-cama

Zapato-manzana

Camisa-zapato

Gato-vaca

Manzana-gato

Pera-vaca

Chancho*-gato

Vestido-pera

Naranja-chancho*

Pera-naranja

Vestido-cama

Silla-mesa

Vaca-chancho*

Zapato-mesa

Vestido-camisa

Manzana-pera

Gato-zapato

Cama-silla

Zapato-vestido

"Chancho equivale a cerdo en España. 
